\title{
INTERPOLATING SEQUENCES IN THE POLYDISC
}

\author{
BO BERNDTSSON, SUN-YUNG A. CHANG AND KAI-CHING LIN
}

\begin{abstract}
Let $H^{\infty}\left(D^{n}\right)$ denote the set of all bounded analytic functions defined on the polydisc $D^{n}$ of $\mathbf{C}^{n}$. In this note, we give a sufficient condition for sequences of points in $D^{n}$ to be interpolating sequences for $H^{\infty}\left(D^{n}\right)$. We also discuss some conditions for interpolation of general domains.
\end{abstract}

Let $C(X)$ be the set of all bounded continuous functions on a compact set $X$ and let $A \subseteq C(X)$ be a uniform algebra equipped with the sup norm. We say that a sequence $\left\{a_{j}\right\}$ of points in $X$ is an interpolating sequence for $A$ if $A \mid\left\{a_{j}\right\}=l^{\infty}$. In other words, $\left\{a_{i}\right\}$ is an interpolating sequence for $A$ if whenever given a bounded sequence $\left\{\alpha_{j}\right\}$, there is a function $f \in A$ such that $f\left(a_{j}\right)=\alpha_{j}$. In the case when $X=D=$ the unit disc in $\mathbf{C}$ and $A=H^{\infty}(D)=$ all the bounded analytic functions on $D$, Carleson proved the following famous theorem (see [3]).

THEOREM (CARLESON). The following are equivalent:

(a) There is a constant $\delta>0$ such that

$$
\prod_{k \neq j}\left|\frac{a_{k}-a_{j}}{1-\bar{a}_{j} a_{k}}\right| \geq \delta>0 \quad \text { for all } j .
$$

(b) $\left\{a_{j}\right\}$ is an interpolating sequence for $H^{\infty}(D)$.

(c) For $k \neq j,\left|\left(a_{k}-a_{j}\right) /\left(1-\bar{a}_{j} a_{k}\right)\right| \geq a>0$ for some $a$, and the measure

$$
\mu=\sum_{j}\left(1-\left|a_{j}\right|\right) \delta_{a_{j}}
$$

is a Carleson measure on $D$, when $\delta_{a_{j}}$ is the Dirac measure at $a_{j}$.

If to each point $a_{j}$ we associate an arc $I_{j}$ in $\partial D$ centered at $a_{j} /\left|a_{j}\right|$ with length $2\left(1-\left|a_{j}\right|\right)$, then the second part of condition (c) indicates that there is a constant $C$ such that

$$
\sum_{I_{j} \subseteq I}\left|I_{j}\right| \leq C|I|
$$

for all $\operatorname{arcs} I$ in $\partial D$. (Here $|E|$ denotes the Lebesgue measure of the set $E$.)

The purpose of this note is to study the analogue of these conditions on the polydisc $D^{n}$ of $\mathbf{C}^{n}$. On the same topic, the readers are referred to earlier works of Kronstadt [7] and E. Amar [1]. Our approach to the problem is quite different from theirs. First we recall some definitions:

Received by the editors January 21, 1986.

1980 Mathematics Subject Classification (1985 Revision). Primary 42B30; Secondary 41A05.

Key words and phrases. Interpolating sequences, Carleson measure, Gleason distance.

The research of the second and third authors was supported in part by NSF grants. 
Definition. Let $p, q$ be two points in $X$. Then the Gleason distance between $p$ and $q$ for the uniform algebra $A$ is defined to be

$$
\rho(p, q)=\sup \left\{|f(p)|: f \in A,\|f\|_{\infty} \leq 1, f(q)=0\right\} .
$$

It is well known that when $X=D=$ the unit disc, $A=H^{\infty}(D), \rho\left(a_{k}, a_{j}\right)$ is equal to $\left|\left(a_{k}-a_{j}\right) /\left(1-\bar{a}_{j} a_{k}\right)\right|$, and when $X=D^{n}=$ the polydisc, $A=H^{\infty}\left(D^{n}\right)$, then

$$
\begin{aligned}
\rho\left(a_{k}, a_{j}\right) & =\max \left(\rho_{1}\left(a_{k}, a_{j}\right), \rho_{2}\left(a_{k}, a_{j}\right), \ldots, \rho_{n}\left(a_{k}, a_{j}\right)\right) \\
& =\max \left(\left|\frac{a_{k}^{1}-a_{j}^{1}}{1-\bar{a}_{j}^{1} a_{k}^{1}}\right|,\left|\frac{a_{k}^{2}-a_{j}^{2}}{1-\bar{a}_{j}^{2} a_{k}^{2}}\right|, \ldots,\left|\frac{a_{k}^{n}-a_{j}^{n}}{1-\bar{a}_{j}^{n} a_{k}^{n}}\right|\right),
\end{aligned}
$$

where $a_{j}=\left(a_{j}^{1}, \ldots, a_{j}^{n}\right) \in D^{n}$.

Again, to each point $a_{j}$ in $D^{n}$ we associate a rectangle $R_{j}$ in the distinguished boundary $T^{n}$ centered at $\left(a_{j}^{1} /\left|a_{j}^{1}\right|, a_{j}^{2} /\left|a_{j}^{2}\right|, \ldots, a_{j}^{n} /\left|a_{j}^{n}\right|\right)$ with side lengths $2\left(1-\left|a_{j}^{1}\right|\right), 2\left(1-\left|a_{j}^{2}\right|\right), \ldots, 2\left(1-\left|a_{j}^{n}\right|\right)$. We are now ready to state our result.

THEOREM 1. Let $D^{n}$ be the polydisc in $\mathrm{C}^{n}$, and let (a), (b), and (c) denote the following statements:

(a) There is a constant $\delta>0$ such that

$$
\prod_{k \neq j} \rho\left(a_{k}, a_{j}\right) \geq \delta>0 \quad \text { for all } j
$$

(b) $\left\{a_{j}\right\}$ is an interpolating sequence for $H^{\infty}\left(D^{n}\right)$.

(c) For $k \neq j, \rho\left(a_{k}, a_{j}\right) \geq a>0$ for some $a$, and there is a constant $C$ such that

$$
\sum_{R_{j} \subseteq \Omega}\left|R_{j}\right| \leq C|\Omega|
$$

for all open sets $\Omega$ in $T^{n}$.

Then (a) implies (b), (b) implies (c). However, the converse for each direction is false.

The second part of $(c)$ is equivalent to the statement that the measure $\mu=$ $\sum_{j}\left|R_{j}\right| \delta_{a_{j}}$ is a Carleson measure on $D^{n}$; see [4].

PROOF OF THEOREM 1 . We will prove the theorem on $D^{2}$, the bidisc in $\mathbf{C}^{2}$, only. The direction (b) $\Rightarrow$ (c) is due to Varopoulos [8]. (Although he has only proved that under assumption (b), $\mu$ is Carleson with respect to rectangles, his methods actually implied that $\mu$ is Carleson with respect to open sets.)

Now we will prove that (a) implies (b). To simplify notations, we denote a point in $D^{2}$ by $(z, w)$ and the sequence by $P_{j}=\left(a_{j}, b_{j}\right)$. We will follow P. Jones's elegant idea [6] in one variable to construct P. Beurling's function $F_{j}(z, w)$ such that $F_{j}\left(P_{k}\right)=\delta_{j, k}$ and such that $\sum_{j}\left|F_{j}(z, w)\right| \leq C$ for all $(z, w)$ in $D^{2}$. It is easy to see that the existence of such $F_{j}$ implies that $\left\{P_{j}\right\}$ interpolates $H^{\infty}\left(D^{2}\right)$. (In fact, these two statements are equivalent; see [5, Chapter VII].) We show first that under condition (a) there are bounded analytic functions $B_{j}$ which take $\left\{P_{k}\right\}$ to $\delta_{j, k}$. 
LEMMA 2. There are $B_{j} \in H^{\infty}\left(D^{2}\right)$ such that $\left\|B_{j}\right\|_{\infty} \leq 1 / \delta$ and $B_{j}\left(p_{k}\right)=$ $\delta_{j, k}$.

PROOF OF LEMMA 2. Indeed, these $B_{j}$ 's are just "Blaschke products." An integer $k$ is said to be in $s_{j}(1)$ if

$$
\rho\left(p_{k}, p_{j}\right)=\rho_{1}\left(p_{k}, p_{j}\right)=\left|\frac{a_{k}-a_{j}}{1-\bar{a}_{j} a_{k}}\right|
$$

and in $s_{j}(2)$ otherwise. Define

$$
B_{j}(z, w)=\frac{\prod_{k \in s_{j}(1)}\left(\frac{-\bar{a}_{k}}{\left|a_{k}\right|} \frac{z-a_{k}}{1-\bar{a}_{k} z}\right) \prod_{k \in s_{j}(2)}\left(\frac{-\bar{b}_{k}}{\left|b_{k}\right|} \frac{w-b_{k}}{1-\bar{b}_{k} w}\right)}{\prod_{k \in s_{j}(1)}\left(\frac{-\bar{a}_{k}}{\left|a_{k}\right|} \frac{a_{j}-a_{k}}{a-\bar{a}_{k} a_{j}}\right) \prod_{k \in s_{j}(2)}\left(\frac{-\bar{b}_{k}}{\left|b_{k}\right|} \frac{b_{j}-b_{k}}{1-\bar{b}_{k} b_{j}}\right)} .
$$

Clearly, $B_{j}\left(p_{k}\right)=\delta_{j, k}$ and $\left\|B_{j}\right\|_{\infty} \leq 1 / \delta$ by condition (a). Next we form P. Beurling's functions. Set

$$
\begin{aligned}
F_{j}(z, w)= & B_{j}(z, w)\left(\frac{1-\left|a_{j}\right|^{2}}{1-\bar{a}_{k} z}\right)^{2}\left(\frac{1-\left|b_{j}\right|^{2}}{1-\bar{b}_{j} w}\right)^{2} \\
& \times \exp \left\{-\frac{1}{4 \log \frac{1}{\delta}} \sum_{\substack{k \in s_{j}(1) \\
\left|a_{k}\right| \geq\left|a_{j}\right|}}\left(\frac{1+\bar{a}_{k} z}{1-\bar{a}_{k} z}-\frac{1+\bar{a}_{k} a_{j}}{1-\bar{a}_{k} a_{j}}\right)\left(1-\left|a_{k}\right|^{2}\right)\right. \\
& \left.-\sum_{\substack{k \in s_{j}(2) \\
\left|b_{k}\right| \geq\left|b_{j}\right|}}\left(\frac{1+\bar{b}_{k} w}{1-\bar{b}_{k} w}-\frac{1+\bar{b}_{k} b_{j}}{1-\bar{b}_{k} b_{j}}\right)\left(1-\left|b_{k}\right|^{2}\right)\right\} .
\end{aligned}
$$

It is readily seen that $F_{j}\left(p_{k}\right)=\delta_{j, k}$. To show that $\sum_{j}\left|F_{j}(z, w)\right| \leq C$, we need to estimate the following sum.

$$
\begin{aligned}
\operatorname{Re} & \left\{\sum_{\substack{k \in s_{j}(1) \\
\left|a_{k}\right| \geq\left|a_{j}\right|}}\left(\frac{1+\bar{a}_{k} a_{j}}{1-\bar{a}_{k} a_{j}}\right)\left(1-\left|a_{k}\right|^{2}\right)+\sum_{\substack{k \in s_{j}(2) \\
\left|b_{k}\right| \geq\left|b_{j}\right|}}\left(\frac{1+\bar{b}_{k} b_{j}}{1-\bar{b}_{k} b_{j}}\right)\left(1-\left|b_{k}\right|^{2}\right)\right\} \\
= & \sum_{\substack{k \in s_{j}(1) \\
\left|a_{k}\right| \geq\left|a_{j}\right|}} \frac{\left(1-\left|a_{j}\right|^{2}\left|a_{k}\right|^{2}\right)\left(1-\left|a_{k}\right|^{2}\right)}{\left|1-\bar{a}_{k} a_{j}\right|^{2}} \\
& +\sum_{\substack{k \in s_{j}(2) \\
\left|b_{k}\right| \geq\left|b_{j}\right|}} \frac{\left(1-\left|b_{k}\right|^{2}\left|b_{j}\right|^{2}\right)\left(1-\left|b_{k}\right|^{2}\right)}{\left|1-\bar{b}_{k} b_{j}\right|^{2}} \\
& \leq 2 \sum_{\substack{k \in s_{j}(1) \\
\left|a_{k}\right| \geq\left|a_{j}\right|}} \frac{\left(1-\left|a_{k}\right|^{2}\right)\left(1-\left|a_{j}\right|^{2}\right)}{\left|1-\bar{a}_{k} a_{j}\right|^{2}}+2 \sum_{\substack{k \in s_{j}(2) \\
\left|b_{k}\right| \geq\left|b_{j}\right|}} \frac{\left(1-\left|b_{k}\right|^{2}\right)\left(1-\left|b_{j}\right|^{2}\right)}{\left|1-\bar{b}_{k} b_{j}\right|^{2}}
\end{aligned}
$$


By the inequality $-\log x \geq 1-x$ for $x>0$ and the equality

$$
1-\left|\frac{a_{k}-a_{j}}{1-\bar{a}_{j} a_{k}}\right|^{2}=\frac{\left(1-\left|a_{j}\right|^{2}\right)\left(1-\left|a_{k}\right|^{2}\right)}{\left|1-\bar{a}_{j} a_{k}\right|^{2}}
$$

we can bound the sum above by

$$
\begin{gathered}
-2 \log \left\{\left(\prod_{k \in s_{j}(1)}\left|\frac{a_{k}-a_{j}}{1-\bar{a}_{j} a_{k}}\right|^{2}\right)\left(\prod_{k \in s_{j}(2)}\left|\frac{b_{k}-b_{j}}{1-\bar{b}_{j} b_{k}}\right|^{2}\right)\right\} \\
\leq 2 \log \frac{1}{\delta}, \quad \text { by condition (a). }
\end{gathered}
$$

Therefore, we have, for $(z, w) \in T^{2}$,

$$
\begin{aligned}
\sum_{j=1}^{\infty}\left|F_{j}(z, w)\right| \leq & \frac{1}{\delta} \sum_{j=1}^{\infty}\left|\frac{1-\left|a_{j}\right|^{2}}{1-\bar{a}_{j} z}\right|^{2}\left|\frac{1-\left|b_{j}\right|^{2}}{1-\bar{b}_{j} w}\right|^{2} \\
& \times \exp \left[\frac{1}{4 \log \frac{1}{\delta}}\left\{-\sum_{\substack{k \in s_{j}(1) \\
\left|a_{k}\right| \geq\left|a_{j}\right|}}\left|\frac{1-\left|a_{j}\right|^{2}}{1-\bar{a}_{k} z}\right|^{2}-\sum_{\substack{k \in s_{j}(2) \\
\left|b_{k}\right| \geq\left|b_{j}\right|}}\left|\frac{1-\left|b_{k}\right|^{2}}{1-\bar{b}_{k} w}\right|^{2}\right\}\right] \\
& \leq \frac{1}{\delta} \sum_{j=1}^{\infty}\left|\frac{1-\left|a_{j}\right|^{2}}{1-\bar{a}_{j} z}\right|^{2}\left|\frac{1-\left|b_{j}\right|^{2}}{1-\bar{b}_{j} w}\right|^{2} \\
& \times \exp \frac{1}{4 \log \frac{1}{\delta}}\left\{-\frac{1}{4} \sum_{k \in s_{j}}\left|\frac{1-\left|a_{k}\right|^{2}}{1-\bar{a}_{k} z}\right|^{2}\left|\frac{1-\left|b_{k}\right|^{2}}{1-\bar{b}_{k} w}\right|^{2}\right\},
\end{aligned}
$$

where $s_{j}=\left\{k: k \in s_{j}(1),\left|a_{k}\right| \geq\left|a_{j}\right|\right\} \cup\left\{k: k \in s_{j}(2),\left|b_{k}\right| \geq\left|b_{j}\right|\right\}$. (We have used the fact that $\left|\left(1-\left|a_{k}\right|^{2}\right) /\left(1-\bar{a}_{k} z\right)\right|,\left|\left(1-\left|b_{k}\right|^{2}\right) /\left(1-\bar{b}_{k} w\right)\right| \leq 2$ for all $(z, w) \in D^{2}$ in getting the last inequality.) Notice that the sets $s_{j}$ have the property that if $k \notin s_{j}$, then $j \in s_{k}$. By letting

$$
c_{j}=\left|\frac{1-\left|a_{j}\right|^{2}}{1-\bar{a}_{j} z}\right|^{2}\left|\frac{1-\left|b_{j}\right|^{2}}{1-\bar{b}_{j} w}\right|^{2} / \log \frac{1}{\delta}
$$

we can complete our proof that (a) implies (b) once we establish the following lemma.

LEMMA 3. Suppose $s_{j}$ are sets of positive integers with the property that if $k \notin s_{j}$ then $j \in s_{k}$ and suppose that $c_{j} \geq 0$. Then

$$
\sum_{j=1}^{\infty} c_{j} \exp \left\{-\sum_{k \in s_{j}} c_{k}\right\} \leq 6 .
$$

ProOF OF LEMMA 3. Let

$$
A_{n}=\left\{j: n-1 \leq \sum_{k \in s_{j}} c_{k}<n\right\} .
$$


Then

$$
\sum_{j=1}^{\infty} c_{j} \exp \left\{-\sum_{k \in s_{j}} c_{k}\right\} \leq e \sum_{n=1}^{\infty}\left(\sum_{j \in A_{n}} c_{j}\right) e^{-n}
$$

To estimate $\sum_{j \in A_{n}} c_{j}$, we notice that

$$
\left(\sum_{j \in A_{n}} c_{j}\right)\left(\sum_{k \in s_{j}} c_{k}\right) \leq n \sum_{j \in A_{n}} c_{j}
$$

On the other hand,

$$
\begin{aligned}
\left(\sum_{j \in A_{n}} c_{j}\right)\left(\sum_{k \in s_{j}} c^{k}\right) & \geq\left(\sum_{j \in A_{n}} c_{j}\right)\left(\sum_{\substack{k \in s_{j} \\
k \in A_{n}}} c_{k}\right) \\
& \geq \frac{1}{2} \sum_{\substack{j \in A_{n} \\
k \in A_{n}}} c_{k} c_{j}, \quad \text { by the property of the } s_{j} \\
& =\frac{1}{2}\left(\sum_{j \in A_{n}} c_{j}\right)^{2}
\end{aligned}
$$

Thus $\sum_{j \in A_{n}} c_{j} \leq 2 n$.

Therefore,

$$
\sum_{j=1}^{\infty} c_{j} \exp \left(-\sum_{k \in s_{j}} c_{k}\right) \leq 2 e \sum_{n=1}^{\infty} n e^{-n} \leq 2 e \int_{0}^{\infty} x e^{-x} d x=2 e \leq 6
$$

This finishes the proof that (a) implies (b).

We give now an example to show that (b) does not necessarily imply (a). (A similar example has also appeared in Kronstadt [7, p. 393].) For simplicity we work with the bi-upper half-plane, $\mathbf{R}_{+}^{2} \times \mathbf{R}_{+}^{2}$, instead of $D^{2}$. Let $\left\{a_{j}\right\}$ be a sequence in $\mathbf{R}_{+}^{2}$ with $a_{j}=j+i, j=0,1,2, \ldots\left\{a_{j}\right\}$ is clearly an interpolating sequence $H^{\infty}\left(\mathbf{R}_{+}^{2}\right)$. Consider the cross product of $\left\{a_{j}\right\}$ with itself, namely, $\left\{\left(a_{k}, a_{j}\right)\right\}_{k, j=0}^{\infty}$, which is an interpolating sequence for $H^{\infty}\left(\mathbf{R}_{+}^{2} \times \mathbf{R}_{+}^{2}\right)$ because of the following trivial observation.

PROPOSITION 4. Suppose $\left\{a_{j}\right\}$ is an interpolating sequence for $H^{\infty}\left(\mathbf{R}_{+}^{2}\right)$, then the sequence $\left\{\left(a_{k}, a_{j}\right)\right\}$ interpolates $H^{\infty}\left(\mathbf{R}_{+}^{2} \times \mathbf{R}_{+}^{2}\right)$.

PROOF OF PROPOSITION 4. The assumption guarantees the existence of P. Beurling's functions $F_{j}(z)$ on $\mathbf{R}_{+}^{2}$ for $\left\{a_{j}\right\}$. Now we simply take $G_{k, j}(z, w)$ $=F_{k}(z) F_{j}(w)$. These $G_{k, j}(z, w)$ are P. Beurling's interpolating functions for $\left\{\left(a_{k}, a_{j}\right)\right\}$. 
However, the sequence $\left\{\left(a_{k}, a_{j}\right)\right\}$ defined above does not satisfy the product condition (a): Let us fix $\left(a_{0}, a_{0}\right)$ and compute

$$
\begin{aligned}
\sum_{k, j} 1 & -\rho^{2}\left(\left(a_{k}, a_{j}\right),\left(a_{0}, a_{0}\right)\right) \\
& \geq \sum_{j} \sum_{k \geq j} 1-\rho^{2}\left(\left(a_{k}, a_{j}\right),\left(a_{0}, a_{0}\right)\right) \\
& =\sum_{j} \sum_{k \geq j} 1-\rho^{2}\left(a_{0}, a_{0}\right)=\sum_{j} \sum_{k \geq j} \frac{4\left(\operatorname{Im} a_{k}\right)\left(\operatorname{Im} a_{0}\right)}{\left|a_{k}-\bar{a}_{0}\right|^{2}} \\
& =4 \sum_{j} \sum_{k \geq j} \frac{1}{k^{2}+2^{2}}=\infty .
\end{aligned}
$$

The example which shows that (c) does not imply (b) is also easy. Take a sequence $\left\{p_{j}\right\}$ in the diagonal of $D^{2}$, namely, $p_{j}=\left(a_{j}, a_{j}\right)$. If (c) were sufficient for (b) then we would have the following sequences of equivalent relationships:

(c)

$$
\left\{\begin{array}{l}
\text { (1) } \rho\left(p_{k}, p_{j}\right)=\left|\frac{a_{k}-a_{j}}{1-\bar{a}_{j} a_{k}}\right| \geq a>0, \quad k \neq j . \\
\text { (2) } \sum_{I_{j} \subseteq I}\left|I_{j}\right|^{2} \leq c|I|^{2} \quad \text { for all arcs } I \text { in } \partial D, \text { where } I_{j}
\end{array}\right.
$$

$\Leftrightarrow\left\{p_{j}=\left(a_{j}, a_{j}\right)\right\}$ interpolates $H^{\infty}\left(D^{2}\right)$

$\Leftrightarrow\left\{a_{j}\right\}$ is an interpolating sequence for $H^{\infty}(D)$.

$$
\Leftrightarrow\left(\mathrm{c}^{\prime}\right) \quad\left\{\begin{array}{l}
(1) \quad \rho\left(a_{k}, a_{j}\right)=\left|\frac{a_{k}-a_{j}}{1-\bar{a}_{j} a_{k}}\right| \geq a>0, \quad k \neq j . \\
(2) \quad \sum_{i_{j} \subseteq I}\left|I_{j}\right| \leq c|I| \quad \text { for all arcs } I \text { on } \partial D .
\end{array}\right.
$$

Now it is clear that conditions (c) and $\left(c^{\prime}\right)$ are not equivalent. To see this, we can simply consider the collection of all dyadic intervals $\left\{I_{k, n}\right\}, I_{k, n}=$ $\left[(k-1) / 2^{n}, k / 2^{n}\right], 1 \leq k \leq 2^{n}, n$ integers, $n=0,1,2,3, \ldots$ on $[0,1]$.

Condition (2) in ( $\left.c^{\prime}\right)$ fails while condition (2) in (c) holds.

Thus we have completed the proof of Theorem 1 .

2. The statement in Theorem 1 is motivated by an analogous result of the firstnamed author in [2], where the interpolating sequence is defined on the unit ball in $\mathbf{C}^{n}$. Thus it is natural to ask the following question: Is there a general, abstract sufficient condition for a sequence to be an interpolating sequence on any uniform algebra? One way to formulate this question may be the following:

PROBLEM. Let $\rho_{X}$ denote the Gleason distance for $H^{\infty}(X)$ for a bounded domain $X \subseteq \mathbf{C}^{n}$. Suppose $\left\{a_{j}\right\} \subseteq X$ satisfies

$$
\prod_{k \neq j} \rho_{X}\left(a_{k}, a_{j}\right) \geq \delta>0 \quad \text { for all } j .
$$

Is $\left\{a_{j}\right\}$ an interpolating sequence for $H^{\infty}(X)$ ?

We have not been able to answer the above question. But it is interesting to observe that the above general problem is related to the interpolating problem on polydiscs in the following way: 
PROPOSITION 5. If in the statement of Theorem 1, we can prove (a) implies (b) for some constant of interpolation defined by

$$
M=\sup _{\left\|\alpha_{j}\right\|_{\infty} \leq 1} \inf \left\{\|g\|_{H^{\infty}}: g \in H^{\infty}\left(D^{n}\right), g\left(a_{j}\right)=\alpha_{j} \text { for all } j\right\}
$$

independent of dimension $n$ ( $M$ depends on $\delta$ ), then for an arbitrary uniform algebra $A \subseteq C(X)$ and a finite sequence $\left\{a_{j}\right\}_{j=1}^{N} \subseteq X$ with

$$
\prod_{k \neq j} \rho_{A}\left(a_{k}, a_{j}\right) \geq \delta>0
$$

( $\rho_{A}$ denotes the Gleason distance function on $\left.A\right)$, we have that $\left\{a_{j}\right\}_{j=1}^{N}$ is an interpolating sequence for $A$ with the constant of interpolation depending on $\delta$. only, and not on the number of points $N$ in the sequence.

Thus, by a normal family argument, this proposition gives an affirmative answer to the problem proposed above, provided that one can prove that the constant of interpolation $M$ for $H^{\infty}\left(D^{n}\right)$ is independent of $n$.

PROOF OF PROPOSITION 5. Let $\left\{a_{j}\right\}_{j=1}^{N}$ be a finite sequence in $X$ satisfying (2). For each pair $\left(a_{k}, a_{j}\right)$ we choose a function $G_{k, j}(z) \in A$ such that $G_{k, j}\left(a_{j}\right)=$ $0,\left|G_{k, j}\left(a_{k}\right)\right| \geq\left(1-1 / 2^{k+j}\right) \rho_{A}\left(a_{k}, a_{j}\right)$ and $\left\|G_{k, j}\right\|_{A} \leq 1$. Now consider the following map:

$$
\mathcal{G}: X \rightarrow D^{N^{2}}, \quad z \rightarrow\left\{G_{k, j}(z)\right\}_{k, j=1}^{N} .
$$

We claim that the map $\mathcal{G}$ essentially preserves the distance between $a_{j}$. Indeed,

$$
\begin{aligned}
\rho_{D^{N^{2}}}\left(\mathcal{G}\left(a_{r}, a_{s}\right)\right) & =\rho_{D^{N^{2}}}\left(\left\{G_{k, j}\left(a_{r}\right)\right\},\left\{G_{k, j}\left(a_{s}\right)\right\}\right) \\
& =\max _{k, j} \rho_{D}\left(G_{k, j}\left(a_{r}\right), G_{k, j}\left(a_{s}\right)\right) \geq\left(1-\frac{1}{2^{r+s}}\right) \rho_{A}\left(a_{r}, a_{s}\right) .
\end{aligned}
$$

Thus,

$$
\prod_{k \neq j} \rho_{D^{N^{2}}}\left(g\left(a_{k}\right), g\left(a_{j}\right)\right) \geq \prod_{k \neq j}\left(1-\frac{1}{2^{k+j}}\right) \rho_{A}\left(a_{k}, a_{j}\right) \geq C \delta \quad \text { for all } j .
$$

Thus, under the assumption of Proposition 5, given any $\left\{\alpha_{j}\right\}_{j=1}^{\infty} \in l^{\infty},\left|\alpha_{j}\right| \leq 1$, we can always interpolate the sequence $\left\{\mathcal{G}\left(a_{j}\right)\right\}_{j=1}^{\infty}$ by some $f \in H^{\infty}\left(D^{N^{2}}\right)$ with $f\left(\mathcal{G}\left(a_{j}\right)\right)=\alpha_{j}$ for all $j$, and with $\|f\|_{\infty}$ independent of $N$. Thus the function $f \circ \mathcal{G}$ is a $H^{\infty}$ function in $A$ with $f \circ \mathcal{G}\left(a_{j}\right)=\alpha_{j}$, which finishes the proof of the proposition.

REMARKS. (1) In the case of the unit ball $B_{n}$ in $\mathbf{C}^{n}$, the proof in [2] indeed indicated that the interpolation constant for $H^{\infty}\left(B_{n}\right)$ is independent of $n$.

(2) An upper bound for the interpolation constant in our proof of (a) $\Rightarrow$ (b) in Theorem 1 is of the order $4^{n} \frac{1}{\delta} \log \frac{1}{\delta}$. But it is not clear at all that our proof gives the best interpolation constant. For example for the sequence $\left\{a_{j}\right\}_{j=1}^{2^{n}} \in D^{n}$, where $a_{j}=\varepsilon_{j} r$ for some $\varepsilon_{j} \in \prod_{1}^{n}\{1,-1\}$ and for some fixed $r<1$, the P. Beurling functions $\left\{F_{j}\right\}$ we have constructed for the sequence has $\left\|\sum_{j=1}^{2^{n}}\left|F_{j}\right|\right\|_{\infty}$ of the order

$$
4^{n} \frac{1}{\delta} \log \frac{1}{\delta} \quad\left(\delta=\left(\frac{2 r}{1+r^{2}}\right)^{2^{n}-1}\right) .
$$


While if we let

$$
g_{+1}(z)=\frac{(z+r)(1+r z)}{2 r\left(1+r^{2}\right)}, \quad g_{-1}(z)=\frac{(r-z)(1-r z)}{2 r\left(1+r^{2}\right)}
$$

for $z \in D$ then the functions $g_{\varepsilon_{j}}\left(z_{1}, \ldots, z_{n}\right)=\prod_{i=1}^{n} g_{\left(\varepsilon_{j}\right) i}\left(z_{i}\right)$ where $\left(\varepsilon_{j}\right)_{i}$ is the $i$-component of $\varepsilon_{j}, 1 \leq i \leq n, 1 \leq j \leq 2^{n}$, also form P. Beurling interpolation functions for the sequence $\left\{a_{j}\right\}$ with $\left\|\sum_{j=1}^{2^{n}}\left|g_{\varepsilon_{j}}\right|\right\|_{\infty} \leq 1 / r^{n}$, which is bounded with upper bound independent of $n$ if we take $r=1-2^{-n / 2}$.

(3) In the proof of Theorem 1, we can also verify (as in Proposition 6 below) that $(\mathrm{a}) \rightarrow$ (c) directly without applying the Varopoulos result (that (b) $\rightarrow(\mathrm{c})$ ). One of the advantages of such a direct proof is that under condition (a), the norm of the Carleson measure can be seen to depend only on $\delta$ (and not on the dimension $n$ ).

PROPOSITION 6. Let $\left\{a_{j}\right\}$ be a sequence in $D^{n}$ satisfying $\prod_{k \neq j} \rho\left(a_{k}, a_{j}\right) \geq$ $\delta>0$. Then there is a constant $c_{\delta}$, depending on $\delta$ only, such that

$$
\sum_{R_{j} \subseteq \Omega}\left|R_{j}\right| \leq c_{\delta}|\Omega|
$$

for all open sets $\Omega$ in $T^{n}$.

PROOF OF PROPOSITION 6. We will prove this proposition on the product of upper half-planes $\left(\mathbf{R}_{+}^{2}\right)^{n}$, where the geometry is simple. In this setting, notice that $\prod_{k \neq j} \rho\left(a_{k}, a_{j}\right) \geq \delta$ implies

$$
\sum_{k} \min _{1 \leq l \leq n}\left\{\frac{y_{k}^{l} y_{j}^{l}}{\left|a_{k}^{l}-\bar{a}_{j}^{l}\right|^{2}}\right\} \leq c_{\delta},
$$

where $a_{j}^{l}=x_{j}^{l}+i y_{j}^{l}$, and $c_{\delta}$ is independent of $n$.

LEMMA 7. Let $\left\{a_{j}\right\}$ be a sequence of points in $\left(\mathbf{R}_{+}^{2}\right)^{n}$ satisfying (**). Then there is a constant $c_{\delta}$, depending on $\delta$ only, such that

$$
\sum_{\left|R_{k}\right| \leq\left|R_{j}\right|}\left|R_{k} \cap R_{j}\right| \leq c_{\delta}\left|R_{j}\right|
$$

PROOF. To prove the lemma it suffices to prove that all the rectangles $R_{j}$ are dyadic. We first observe that, in the case of one variable, if $I_{k} \subseteq I_{j}$ or $I_{j} \subseteq I_{k}\left(I_{j}\right.$ is the interval associated to the point $a_{j}$ in $\mathbf{R}_{+}^{2}$ ), then

$$
1-\rho^{2}\left(a_{k}, a_{j}\right)=\frac{4 y_{k} y_{j}}{\left|a_{k}-\bar{a}_{j}\right|^{2}} \approx \frac{\min \left(y_{k}, y_{j}\right)}{\max \left(y_{k}, y_{j}\right)}, \text { where } a_{j}=x_{j}+i y_{j} \text {. }
$$

Thus $(* *)$ implies that

$$
\sum_{R_{k} \cap R_{j} \neq \varnothing} \min _{l}\left\{\frac{\min \left(y_{k}^{l}, y_{j}^{l}\right)}{\max \left(y_{k}^{l}, y_{j}^{l}\right)}\right\} \leq c_{\delta} .
$$

On the other hand, the estimate in Lemma 7 is equivalent to

$$
\sum_{\substack{R_{k} \cap R_{j} \neq \varnothing \\\left|R_{k}\right| \leq\left|R_{j}\right|}} \prod_{l=1}^{n} \min \left(y_{k}^{l}, y_{j}^{l}\right) \leq c_{\delta} \prod_{l=1}^{n} y_{j}^{l}
$$


Dividing both sides of this inequality by $\prod_{l=1}^{n} y_{j}^{l}$, to prove Lemma 7 we need only to verify that

$$
\text { if } \prod_{l=1}^{n} y_{k}^{l} \leq \prod_{l=1}^{n} y_{j}^{l}, \quad \text { then } \prod_{l=1}^{n} \frac{\min \left(y_{k}^{l}, y_{j}^{l}\right)}{y_{j}^{l}} \leq \min _{l}\left\{\frac{\min \left(y_{k}^{l}, y_{j}^{l}\right)}{\max \left(y_{k}^{l}, y_{j}^{l}\right)}\right\}
$$

which is an inequality quite elementary to check.

To finish the proof of Proposition 6 , we write $\Omega=\bigcup_{i} R_{j}$. Summing both sides of the inequality in Lemma 7 in $j$ yields

$$
\sum_{j} \sum_{\left|R_{k}\right| \leq\left|R_{j}\right|}\left|R_{k} \cap R_{j}\right| \leq c_{\delta} \sum_{j}\left|R_{j}\right|
$$

The left-hand side is greater than or equal to $\sum_{k, j}\left|R_{k} \cap R_{j}\right|$. Writing it in integral form, we obtain

$$
\int\left(\sum_{j} \chi_{R_{j}}\right)^{2}=\sum_{k, j}\left|R_{k} \cap R_{j}\right| \leq c_{\delta} \sum_{j}\left|R_{j}\right|=c_{\delta} \int\left(\sum \chi_{R_{j}}\right) .
$$

Applying Hölder's inequality we then have

$$
\sum_{j}\left|R_{j}\right|=\int \sum \chi_{R_{j}} \leq c_{\delta}\left|\bigcup_{i} R_{j}\right|=c_{\delta}|\Omega| .
$$

The proof of Proposition 6 is thus complete.

\section{REFERENCES}

1. E. Amar, Interpolation dans le polydisque de $\mathbf{C}^{n}$, Analyse Harmonique Orsay, 207, 1976.

2. B. Berndtsson, Interpolating sequences for $H^{\infty}$ in the ball, Nederl. Akad. Wetensch. Indag. Math. 88 (1985).

3. L. Carleson, An interpolation problem for bounded analytic functions, Amer. J. Math. 80 (1958), 921-930.

4. S.-Y. A. Chang, Carleson measures on the bidisc, Ann. of Math. 109 (1979), 613-620.

5. J. Garnett, Bounded analytic functions, Academic Press, New York, 1981.

6. P. Jones, $L^{\infty}$-estimates for the $\bar{\partial}$ - problem in a half-plane, Acta Math. 150 (1982), 136-152.

7. E. Kronstadt, Interpolating sequences in polydiscs, Trans. Amer. Math. Soc. 199 (1974), 369-398.

8. N. Th. Varopoulos, Sur un problème d'interpolation, C. R. Acad. Sci. Paris Ser. A 274 (1972), 1539-1542.

Department of Mathematics, University of Göteborg, S-142, 96 Göteborg, SWEDEN

Department of Mathematics, University of California, los Angeles, CaliFORNIA 90024

Department of Mathematics, University of Wisconsin-Madison, Madison, WISCONSIN 53706 